\title{
Milling versus Sawing: Comparison of Temperature Elevation and Clinical Performance During Bone Cutting
}

\author{
A. Malvisi, P. Vendruscolo, F. Morici, S. Martelli, M. Marcacci. \\ Andrea Malvisi Lab. Biomeccanica via di Barbiano 1/10, 40136 Bologna, Italy. \\ Tel.:+390516366520 - Fax:+39051583789 \\ E-mail: a.malvisi@biomec.ior.it - URL: http://www.ior.it/biomec/
}

\begin{abstract}
A fundamental requirement for the success of an implant process is the quality of bone resection, mainly related to temperature elevation during cutting and to the accuracy of the resection. Existing surgical saws have largely been investigated, but appear still unable to maintain the temperature elevation within acceptable parameters. In this paper we analyse an alternative approach to realising large bone resections, studying the performance of the milling technique. The temperature elevation during milling is carefully analysed with different surgical tools, and an overall estimation of the accuracy and usability of this technique is discussed. The results of this study prove that milling can be used without the need for cooling procedures $\left(\mathrm{T}<46^{\circ} \mathrm{C}\right)$, within an acceptable surgical time ( $\sim 4 \mathrm{~min}$ for knee resections), and with negligible fatigue to the user.
\end{abstract}

\section{Introduction}

In orthopaedic surgery large resections are usually performed using surgical saws. This technique involves the use of oscillating air-powered saws, with special materials for sterilisation and a special design for fast and ergonomic use. Modern high-speed cutting machines have a shortened operating time, but a major drawback of such equipment is the tissue damage due to the release of energy. Resulting resections have a satisfactory quality in most cases, but some problems remain in controlling the final accuracy of the cut and the temperature elevation during bone machining.

Several analyses have shown that commercially available surgical saws are statistically unable to constrain the temperature elevation below $44^{\circ} \mathrm{C}$ to $47^{\circ} \mathrm{C}$, which is the critical limit range for heat-induced bone necrosis [1] [2]. Despite the influence of mechanical and geometrical blade features on the saws' performance [1] [3] [4] [5], the temperature registered during bone sawing is generally very high and can reach even $450^{\circ} \mathrm{C}$ [5]. Only internally cooled saw prototypes [6] can lower the cutting temperature to a reasonable level in order to keep a normal osteointegration and prevent anomalous bone modelling.

The attempt to overcome the temperature rise during cutting led us to investigate a different approach to realising bone resections, based on the use of a high-speed power milling tool installed at the end of a robot arm with five degrees of freedom.

Our study, performed on animal limbs, investigates the performance and usability of this technique to realise knee resections. 
In this study we have considered the other critical factors influencing the possible use of the milling technique for surgical interventions: firstly the temperature elevation and propagation during cutting, and secondly the time requirements and the usability in a surgical environment. Therefore this work completes an original investigation on a new technique for bone resections in orthopaedic interventions.

\section{Materials \& Methods}

We studied 15 knees defrosted, a few weeks the subject animal was killed in the normal way, to insure a sufficient level of hydration. The limbs were locked in a fixed position with a clamping device. Bones appeared, on manual inspection, to be within the normal range of individual variability of shape, size, bone density, and cartilage thickness, and had an average resection surface of $1925.5 \mathrm{~mm}^{2}$. To simulate the in vivo conditions bones were heated to a temperature of between $34^{\circ} \mathrm{C}$ and $37^{\circ} \mathrm{C}$ at the bone core, by a heating element located far enough from the milled surface (more than $450 \mathrm{~mm}$ ) not to be affected by the temperature changes produced by the machining.

The temperature measured at the surface of the bone before the beginning of the milling was from $1^{\circ} \mathrm{C}$ to $3^{\circ} \mathrm{C}$ lower than the temperature of the bone core, as in real conditions.

The room temperature was maintained at between $23^{\circ} \mathrm{C}$ and $25^{\circ} \mathrm{C}$.

The measuring instrumentation consisted of two thermocouples: an E-type (nickelchromium vs. copper-nickel) thermocouple specially shaped for penetration probes with a measurement range of $0-250^{\circ} \mathrm{C}$, and a J-type (iron vs. copper-nickel) exposed junction thermocouple with a measurement range of $20-700^{\circ} \mathrm{C}$.

Both devices were used to monitor the bone temperature: the E-type thermocouple was set $1 \mathrm{~mm}$ under the cut and recorded the temperature of the milled surface, while the J-type was set $45 \mathrm{~mm}$ under the cut and recorded the temperature of the core bone (Fig. 1).

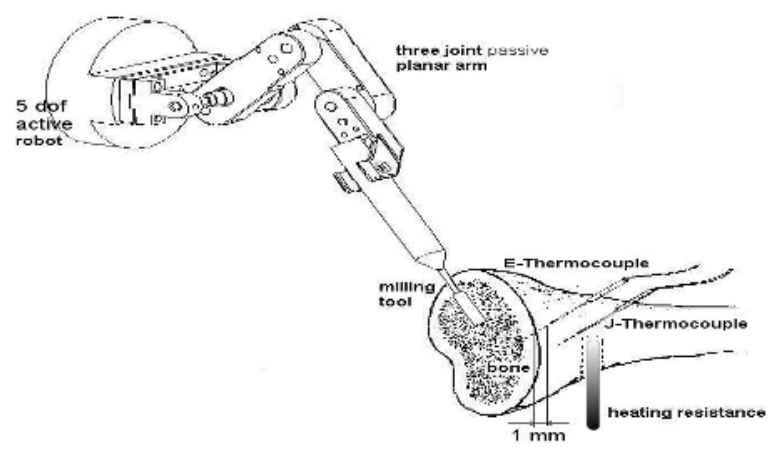

Fig. 1. (a) Experimental setup and equipment. The robot was used to localize both the resection plane and the thermocouples holes. E-type measures surface temperature, J-type measures core bone temperature. The cutting was executed manually using the planar jig. 
The accurate preparation of the holes for the two thermocouples was guaranteed by the use of a robot as location pointer, to drill the holes and also to hold a planar guide for the milling machine in the planned position. The position accuracy of the experiment set-up was $\pm 0.4 \mathrm{~mm}$. The two thermocouples were manually lodged in the relevant holes and cemented with a special thermo-conductive compound (Tech Spray ${ }^{\mathrm{TM}}$ Silicon Free Heat Sink Compound) to fix their position, assure good thermal contact between the bone and the device, and to prevent air infiltration into the hole. Voltage values were read on the junction of the thermocouple every $0.25 \mathrm{sec}$ using an HP 34970A Data Acquisition/Switch unit equipped with a built-in cold reference junction, to monitor precisely the variations of temperature as a function of time.

The milling was carried out using MIDAS REX (Forth Worth, TX, USA) standard dissecting tools with a rotation velocity of between 70,000 and 100,000 rpm. During the tests no measures were taken to cool the milled area.

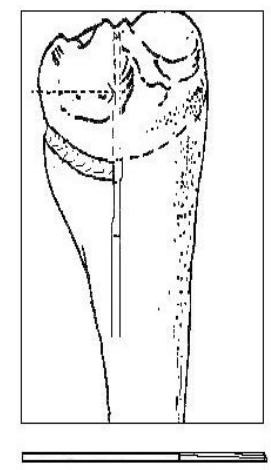

(a)

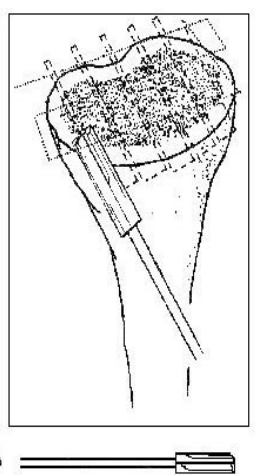

(b)

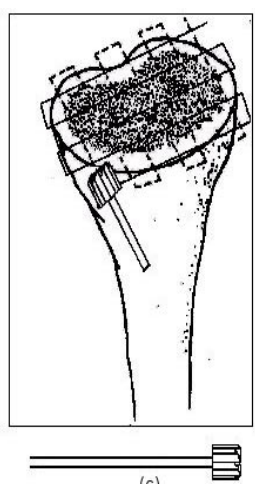

(c)

Fig. 2. Milling tools and relative cutting trajectory to realize a planar resection. The cutting trajectory is exemplified for the tibia cut. (a) M10 (b)M16 (c)M15

We studied 3 types of pneumatic milling cutters with different shapes. In order to exploit their typical features and optimize the quality of the final resections they were used with different attitudes and trajectories for carrying out knee resections:

- M10 tool: diameter $2.5 \mathrm{~mm}$, length $40.5 \mathrm{~mm}$, thin body with blade located along the body following a spiral path and no blade in the frontal region (FIG2 (a)).

- M16 tool: diameter $6.5 \mathrm{~mm}$, length $45.0 \mathrm{~mm}$, tall body with blade located in front and lateral regions, used orienting the blade axis in the AP direction (FIG2 (b)).

- M15 tool: diameter $12.5 \mathrm{~mm}$, length $18.5 \mathrm{~mm}$, slim body with blade located in front and lateral region, used orienting the blade axis in the AP direction (FIG2(c)).

The three mentioned milling tools were evaluated in independent experiments. Each tool was tested on 5 tibia and 5 femurs to realise only one complete sets of resections for knee prosthesis ( 5 planes on femur and one on tibia), as recommended by HOWMEDICA, to get correct performances. Resection were realised manually by the surgeon, using the milling machine on the plane constrained by the robotic jig.

The temperature was monitored during and after each cut until cooling process was over. 
The time needed to finish resections was measured by an external observer.

After each test the surgeon filled in a form prepared for the ergonomic estimation of the tool including a subjective evaluation of the feasibility of the access, the amount of debris, and the fatigue during the task.

\section{Results}

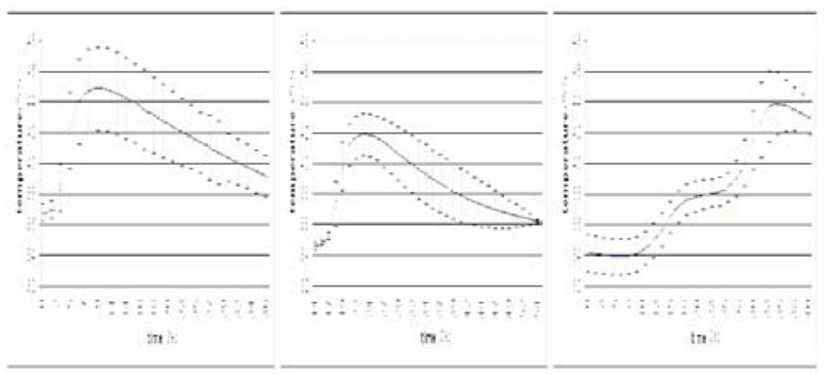

Fig. 3. Bone temperature ( $1 \mathrm{~mm}$ under the surface) during milling. The profile is the mean value for 5 knees and bars show 95\% confidence interval (a) M10 (b)M16 (c)M15 curve.

The temperature behaviour during milling is reported in Fig. 3 for the different tools, as an average on 5 knees. For a clearer comparison of the tools' performance the temperature profile is reported for the tibial cut where the time needed for the complete resection was very similar $( \pm 1 \mathrm{sec}$.) for all knees and did not require normalisation. The temperature profiles for the 5 femoral cuts are similar, with different time ranges according to the resection size.Fig. 4 reports the time needed to perform a complete milling on an average resection surface of $3260 \mathrm{~mm}^{2}$ with the three tools.

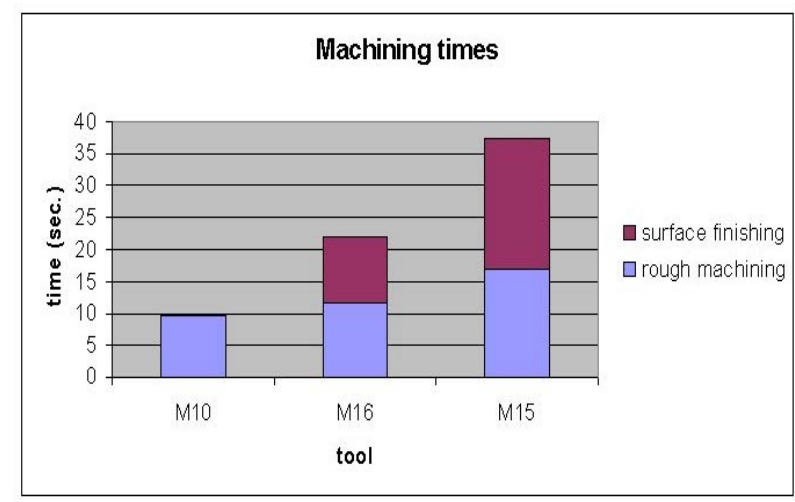

Fig. 4. Milling time for the different tools. The reported time for each tool is computed as the mean value of the tibial resection on 5 knees with an average resection size of $3260 \mathrm{~mm}^{2}$. 
The above mentioned parameters(mean cooling rate, maximum temperature during the milling, milling time) are the basics of the comparison of the three milling tools.

\section{Discussion}

Our results have shown the potential benefits deriving from a milling technique instead of sawing.

All main parameters (time, temperature, amount of chips, surface roughness and flatness, user satisfaction) are satisfactory and, except for the resection time, better than those collected in the literature about sawing techniques.

In particular the temperature of the cortical bone $1 \mathrm{~mm}$ under the cut surface was always lower than $46^{\circ} \mathrm{C}$ (at a core bone temperature of $36^{\circ} \mathrm{C}$ ) for all tools without the use of cooling techniques, and this represents an improvement with respect to sawing [5]. The temperature elevation during bone machining appeared slightly sensitive to individual variation in bone size and density, it was roughly proportional to the resection area and reached the maximum values on the tibial cut. The measured temperature certainly depends on the location of the measuring device. Variations in the temperature are here measured $1 \mathrm{~mm}$ from the milled surface, which showed a significant temperature elevation curve

The time necessary to mill knee resection is usually higher than the time necessary for sawing, because milling needs time consuming trajectories of the tools and often repeated machining of the surface. However it is largely under the critical exposure time for bone tissue injury [7] for all the tools. Therefore the global temperatureexposition rate of milling is better than sawing.

A more accurate analysis of the reported result should take into account the different performance of the tools tested for total knee replacement, which can be important to optimize specific applications.

We can notice that the highest temperature produced by the M10 tool with respect to the M15 and M16 is also related to the presence of chips that are retained near the cut bone, and are known to contain $60-70 \%$ of the total heat produced in metal-cutting operations [8] and commonly extended to bone cutting. On the contrary when the M15 and 16 are used the chips are «vaporized» and only a reduced part of them falls into the surgical area. Moreover the M10 mill is not exposed to the airflow with a consequent loss of cooling effect, and in fact its cooling rate is twice as slow as that of the M15 and M16. We also expect that the M15 and M16 temperature results gain an advantage in the in-vitro experiments, because of the absence of soft tissue making the dispersion of chips produced easier. All these factors explain why the bone temperature reached using the M10 is higher than that using the M15/M16.

On the other side the resecting time with the M10 is much shorter than with the M15 and M16, because the absence of a rough phase for the M10 tool significantly reduces the requested time.

However it can be noticed that Fig. 4 also shows a significant difference in the time required to finish resections for M15 and M16 tools.

This difference is linked to the mode of employment of each tool and its geometrical and mechanical features. 
For the M16 tool the reduced frontal section and a more noticeable frontal rake angle, reduced bending effects, allowing a resection thickness equal to tool diameter and resulted in faster and easier use with compared to the M15, although the resection trajectories are similar.

The M15 has a larger frontal section than the M16 and did not work properly if the resection thickness was greater than $75 \%$ of its diameter, because the forces generated by the friction reduce dramatically the penetration factor of the frontal blade and required more effort by the user to counter bending effect and vibrations. As a consequence rough machining with the M15 tool needed more time than with the M16. Moreover the reduced lateral section of the M15 with respect to the M16 implies a larger number of passages and therefore increase the time for the refining phase, and produces the presence of an anomalous feature in the temperature graph of M15, with respect to the graph of M10 and M16: two temperature peaks, one for the rough machining and one for the refining.

In summary these results showed that the M10 is the best mill for TKR, because its temperature-exposition rate is optimal.

It should be noticed that the general recommendation for the drill process is to use a low feed rate [9] [10], but with our prototype system, we have not reported any drawbacks using milling techniques with tool speed at $100000 \mathrm{rpm}$. By contrast, we have found advantages using a higher feeding rate in accordance with some authors [11] [12]. The M10 performance results were the best and only chip production was worse than for the M15 and M16. As a general consideration, it can be noticed that during milling the surface remains polished, and chips do not accumulate between bone and blade like in sawing, but are dispersed by rotational movement around the working area.

Although further experiments with human bones should be performed in future, the used technique needed very little training ( $2 / 3$ sessions before optimal confidence).

\section{Conclusions}

In this paper we have analysed the temperature elevation and the usability of the milling technique for performing bone resections (in particular, in total knee replacement).

Our results show that all tested tools are able to finish any resection on tibia and femur (from $1320 \mathrm{~mm}^{2}$ to $4960 \mathrm{~mm}^{2}$ ) with the temperature of the cortical bone, $1 \mathrm{~mm}$ under the cut, lower than $46^{\circ} \mathrm{C}$ without using cooling techniques. Moreover the exposition time is largely under the critical value for bone tissue injury and the surgeon's degree of satisfaction is good. These results appear much better than those obtained with commercial saws or even new prototypes proposed in literature. Even if further investigation is probably necessary to evaluate the histological consequences of milling, the reported results provide encouraging indications for a possible introduction of new power tools and the milling technique into orthopaedic surgical practice as an alternative to standard equipment. The use of this technique can improve the overall quality of bone resection in TKR [13], but also reduce the risk of necrosis and could be used without the need for a cooling device, which is, at present, mandatory. 
For TKR In particular, the tested high speed tools have also shown the benefit of reduced friction and control force. The results indicated that the M10 was the most suitable tool because it was simpler and faster to use than the M15 and M16 and still provided an acceptable temperature elevation during milling.

The use of this tool and this cutting technique can be particularly suitable for robotassisted TKR because it has low vibrating feedback on the robot interface, negligible force control and does not require additional devices (such as milling methods or change of tool/approach).

\section{Bibliography}

[1] Krause, W.R., Bradbury D.W., Kelly J.E., Temperature elevations in orthopaedic cutting operations, J. Biomechanics, Vol. 15 No. 4, pp. 267-275 1982.

[2] Larsen S.T. , Lyd L. Temperature elevation during knee arthroplasty, Acta Orthop Scandinava , 1989; 60(4 ): 439-442

[3] Klip EJ., Sawing in bone., Report no. 1, Departement of orthopaedic Surgery and the Institute of Technology, Linkoping, Sweden, 1976.

[4] Krause, W.R., Bone cutting: Mechanical and thermal effects. Proc. Bull. Hosp. Joint Dis. $38,5-7,1977$.

[5] Larsen S.T. , Lyd L., Lindstrand, A. Temperature influence in different orthopaedic saw blades, J of arhoplasty, Vol. 7 No. 1, march 1992.

[6] Larsen S.T. , Lyd L., Lindstrand, A. On the problem of heating generation in bone cutting, J Bone and Joint Surgery, vol. 73-B, No. 1, Jan. 1991

[7] Eriksson AR, Albrektsson T, Albrektsson B, Heat caused by drilling cortical bone: temperature measured in vivo in patients and animals, Acta Orthop Scand, 1984; 55:629-31.

[8] Schmidt A.O., Heat in metal cutting p.128 In Machining Theory and practice. American Society of Metals, Cleveland, 1950

[9] Tetsch P. Development of raised temperature after osteotomies, J Maxillofac Surg, 1974; 2 (2-3): 141-145.

[10] Lavelle C., Wedgwood D. Effect of internal irrigation on frictional het generated from bone milling, J Oral Surg 1980, 38 (7): 499-503

[11] Matthews LS, Hirsh C. Temperature measured in human cortical bone when drilling, J Bone Joint Surg, 1972; 54(2):297-308.

[12] Krause W, Bradbury DW, Kelly JE, Lunceford EM. Temperature elevations in orthopaedic cutting operations, J Biomech, 15:267, 1982

[13] Fadda M., Marcacci M., Toksvig-Larsen S., Wang T., Meneghello R.: Improving accuracy of bone resections using robotics tool holder and a high speed milling cutting tool. Journal of Medical Engineering \& Technology,22(6), 280-284, November/December 1998. 